\title{
Time-Dependent Fluorescence Spectroscopy to Quantify Complex Binding Interactions
}

\author{
Samuel P. Bernhard, Candace K. Goodman, Erienne G. Norton, Daniel G. Alme, C. Martin Lawrence, \\ and Mary J. Cloninger*
}

Cite This: ACS Omega 2020, 5, 29017-29024

Read Online

\section{ACCESS | Lill Metrics \& More | 回 Article Recommendations ｜（） Supporting Information}

ABSTRACT: Measuring the binding affinity for proteins that can aggregate or undergo complex binding motifs presents a variety of challenges. In this study, fluorescence lifetime measurements using intrinsic tryptophan fluorescence were performed to address these challenges and to quantify the binding of a series of carbohydrates and carbohydrate-functionalized dendrimers to recombinant human galectin-3. Collectively, galectins represent an important target for study; in particular, galectin-3 plays a variety of roles in cancer biology. Galectin-3 binding dissociation constants $\left(K_{\mathrm{D}}\right)$ were quantified: lactoside $(73 \pm 4 \mu \mathrm{M})$, methyllactoside (54 $\pm 10 \mu \mathrm{M})$, and lactoside-functionalized $\mathrm{G}(2), \mathrm{G}(4)$, and G(6)-PAMAM dendrimers $(120 \pm 58 \mu \mathrm{M}, 100 \pm 45 \mu \mathrm{M}$, and $130 \pm 25 \mu \mathrm{M}$, respectively). The chosen examples showcase the widespread utility of time-dependent fluorescence spectroscopy for determining binding constants, including interactions for which standard methods have significant limitations.

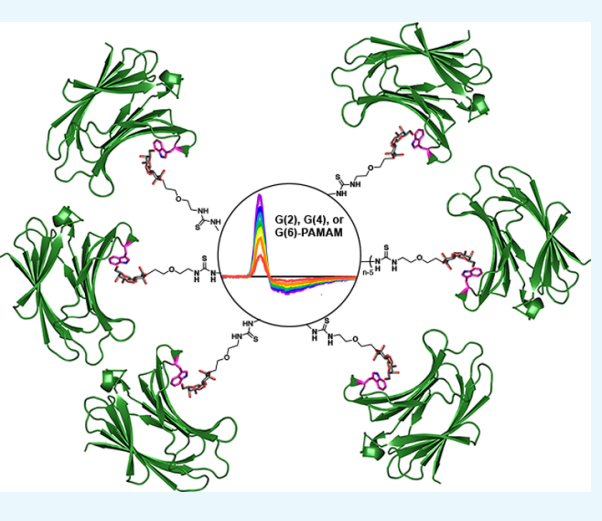

\section{INTRODUCTION}

Protein-ligand binding interactions are currently measured by many different methods. ${ }^{1,2}$ Some of the most common methods include microcalorimetry, fluorescence intensity, absorbance intensity, fluorescence anisotropy, surface plasmon resonance, and the enzyme-linked immunosorbent assay (ELISA). However, many important interactions such as protein-carbohydrate interactions are low-affinity associations that are difficult to measure accurately with commonly available methods. Low protein solubility and protein aggregation at higher concentrations also often complicate measurements of ligand binding. ${ }^{3}$

Fluorescence is a preferred method for monitoring binding interactions because of its speed and sensitivity. ${ }^{4,5}$ Binding is often readily detected by measuring the change in fluorescence intensity at the monitored wavelength for side chains such as tryptophan. 6 , When changes in fluorescence intensity can be observed for the existing protein side chains, then the appendage of an additional fluorescence label is unnecessary. ${ }^{8}$

For systems that undergo very small changes in fluorescence upon binding, fluorescence lifetime (rather than equilibrium) measurements can be invaluable. However, the conventionally longer acquisition times and complex data analysis of fluorescence decay approaches such as time-correlated single photon counting (TCSPC) $)^{4}$ have, in the past, made it more difficult to obtain time-dependent spectra. In contrast, Direct Waveform Recording technology (Fluorescence Innovations) is capable of collecting time-resolved data in a fraction of the time of traditional systems. This technique allows decay curves to be treated as a type of spectrum, resulting in a simplified and accelerated analysis process that works well for fluorescence lifetime measurements with both intrinsic fluorescent groups and appended fluorescent dye labels. Thus, in principle, direct waveform recording makes the fluorescent lifetime technique more practical and accessible for protein binding studies. ${ }^{9}$ In practice, however, this has yet to be widely adopted for the routine determination of dissociation constants.

The binding of carbohydrates to galectin-3 serves to exemplify the advantages of the fluorescence lifetime methodology for measuring low-affinity binding interactions. Galectin3 is a $\beta$-galactoside binding protein with a shallow binding pocket for carbohydrate binding and an $\mathrm{N}$-terminal domain for protein aggregation. ${ }^{10,11}$ Binding to carbohydrates plays an important role in cancer progression, ${ }^{12-14}$ but binding interactions can be difficult to study with galectin-3. Individual association constants for simple mono- and disaccharides with galectin-3 are weak, and the protein aggregates when approaching an oligomeric carbohydrate-functionalized system. ${ }^{15}$ Because of the inherent difficulty of studying these galectin-3 binding interactions, a wide range of dissociation constants for carbohydrate binding to this protein have been

Received: July 16, 2020

Accepted: October 23, 2020

Published: November 6, 2020 
reported. The reported values of the dissociation constants $\left(K_{\mathrm{D}}\right)$ for the binding of galectin-3 to galactose- $\beta 1,4$-glucose (lactose), for example, range from $16^{16}$ to $860 \mu \mathrm{M}^{17}$ and include the values 55, ${ }^{18} 90,{ }^{19} 130,{ }^{20} 140,{ }^{21}$ and $618^{22} \mu \mathrm{M}$. In addition, the reported $K_{\mathrm{D}}$ of methyl-(Gal- $\beta 1,4-\mathrm{Glc}$ ) (methyllactose) is $224 \mu \mathrm{M}^{23}$ This span in reported $K_{\mathrm{D}}$ values is a testament to the challenge of accurately measuring weak associations between proteins that are difficult to study and their low affinity but important ligands.

Previously reported methods to quantify galectin-3 binding affinity have employed calorimetry, ${ }^{17,24,25}$ surface plasmon resonance (SPR), ${ }^{18} \mathrm{NMR},{ }^{20}$ fluorescence intensity measurements, and competitive fluorescence polarization. ${ }^{23,26}$ Some of these methods including calorimetry, NMR, and fluorescence techniques are performed in solution, while SPR experiments and ELISAs rely on surface-bound ligands, receptors, or inhibitors. Isothermal titration microcalorimetry and NMR must be performed at relatively high concentrations of protein (100 $\mu \mathrm{M})$, while fluorescence measurements including fluorescence polarization and the fluorescence lifetime measurements described here can be performed at much lower concentrations $(4.3 \mu \mathrm{M}$ in this experiment, hundred nanomolar in other time-dependent fluorescence measurements, and even low nanomolar concentrations in fluorescence polarization experiments). ${ }^{26-29}$ Fluorescence intensity measurements can be quite successful ${ }^{19}$ but are much more susceptible than the fluorescence lifetime measurements to variations caused by inner filter effects, laser power fluctuations, and concentration discrepancies because of imprecise pipetting which can produce inaccurate results if not considered.

All of these methods have some advantages, and comparisons of the relative association strengths of different ligands when they are determined using the same type of assay are meaningful. However, effects including those mentioned above such as concentration and surface interaction can make it difficult to obtain meaningful absolute values of $K_{\mathrm{D}}$ that can be compared across assay types.

Because galectin-3 has weak monovalent binding interactions with simple mono- and disaccharides and readily aggregates in the presence of carbohydrate-functionalized macromolecules, this protein is ideal for showcasing the utility of time-dependent fluorescence spectroscopy to determine even difficult to measure binding constants. Here, fluorescence lifetime binding studies using galectin-3 with lactoside, methyllactoside, and a series of biomimetic multivalent lactose-functionalized glycodendrimers are reported. These values should serve as unbiased solution-based values to which values obtained from other assays could be benchmarked. Of course, the reported fluorescence lifetime methodology also works well for more straightforward ligand-receptor binding interactions that are not complicated by issues such as aggregation, solubility, or low affinity and can therefore be widely utilized.

\section{RESULTS AND DISCUSSION}

Galectin-3 Binding. The carbohydrate recognition domain (CRD) is a 130 amino acid $\beta$-sandwich composed of residues 112-250 (Figure 1). ${ }^{30,31}$ The S-face of the CRD exhibits a channel capable of binding a range of galactoside-based ligands. Galectin-3 contains three tryptophan residues including a key tryptophan (W181) that mediates a $\mathrm{C}-\mathrm{H} / \pi$ interaction in the canonical S-face binding site (Figure 1,

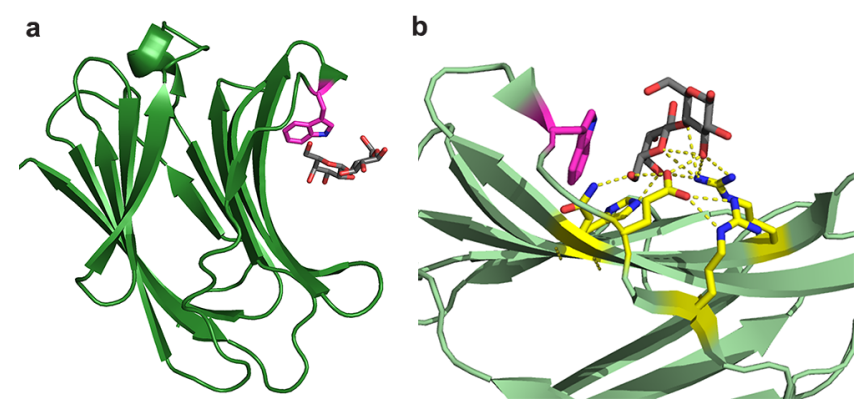

Figure 1. (a) Crystal structure of galectin-3 CRD, shown in green, with lactose in the canonical binding pocket. The environment around the key tryptophan (W181), shown in magenta, is altered by ligand binding. (b) Hydrogen bonds between lactose and H158, R162, N174, E184, and R186 galectin-3 residues, yellow, are shown.

PDBID: $3 \mathrm{ZSJ}^{32}$ ). The other two tryptophan residues are located early on the $N$-terminus (W22 and W26) and do not participate in specific binding interactions. ${ }^{32,33}$

Complex binding events that involve multiple accessible binding sites and potential protein oligomerization, such as those described here, can be modeled by eq 1 .

$$
F_{\mathrm{b}}=B_{\max }\left(\frac{[L]^{n}}{K_{\mathrm{D}}+[L]^{n}}\right)
$$

In this equation, $F_{\mathrm{b}}$ is the fraction of protein present in the bound state, $[L]$ is the concentration of the ligand available in solution, and $B_{\max }$ is the maximum specific ligand binding extrapolated for an extremely high concentration of the ligand. ${ }^{34,35} K_{\mathrm{D}}$ is the dissociation constant, and $n$ is the Hill coefficient. A key feature of this analysis is that observed waveform changes continue with increasing lactose concentration up to a point and then stop, indicating that binding saturation has occurred. Values of $F_{b}$ plotted for each concentration were the coefficients obtained by fitting each waveform to a linear combination of unbound and saturated galectin-3 waveforms. As described in the Supporting Information, no waveform is explicitly determined for the saturated state; instead, the values associated with a saturated waveform are calculated.

The Hill coefficient, $n$, acts in this equation to account for complex stoichiometry and cooperativity. When $n=1$, this indicates that galectin-3 is binding a single carbohydrate in a noncooperative manner. Values greater than one indicate that galectin-3 is involved in more complex binding interactions that may include nonspecific binding, complex stoichiometries, or positive cooperativity. Hill coefficient values less than one indicate negative cooperativity. ${ }^{36}$

Under conditions where a single ligand interacts with a single receptor, as is the case for lactose and methyllactose, data can be appropriately modeled by eq 1 but with the Hill coefficient set to $n=1$. The multivalent behavior of galectin-3, including its capacity to oligomerize, is widely accepted. ${ }^{37-40}$ Therefore, allowing the Hill coefficient to be calculated as a noninteger value is also important to approximate the known nature of galectin-3 in multivalent binding scenarios. Data obtained using both fit models are reported as appropriate. Standard deviations are reported to represent the accuracy of the value determined by the curve fit. ${ }^{35}$

Lactose is an important ligand for study because it is structurally similar to endogenous galectin-3 epitopes, and although it has been explored extensively in the literature, there 

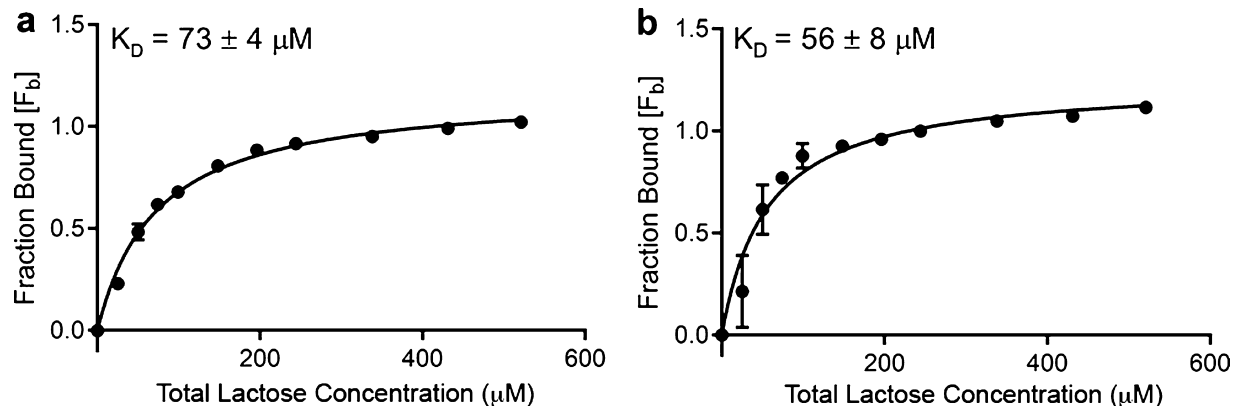

Figure 2. (a) Binding curve for lactose and GST-purified galectin-3. $B_{\max }=1.18$. (b) Binding curve for lactose and His-tagged galectin-3. $B_{\max }=$ 1.26. The concentration of galectin-3 is $4.3 \mu \mathrm{M}$. Error bars represent the standard deviation arising from triplicate repetitions of the assay.

is significant variation in reported binding constants because of the complexity of the system and the challenge of the measurement $(16-860 \mu \mathrm{M}) .^{16-22}$ Figure 2a shows the binding curve and corresponding $K_{\mathrm{D}}$ for lactose binding to GSTpurified galectin- 3 , as determined by the fluorescence lifetime experiment. The $K_{\mathrm{D}}$ was determined to be $73 \pm 4 \mu \mathrm{M}$ or toward the lower end of the reported range of values. The $K_{\mathrm{D}}$ for His-tagged galectin-3 with lactose was $56 \pm 8 \mu \mathrm{M}$ (Figure $2 \mathrm{~b})$. As expected, these binding constants are very nearly the same; with error bars, the values are only $5 \mu \mathrm{M}$ from overlapping. This indicates that the differences in purification protocols, including the addition of the six amino acid residue histidine sequence appended to the $\mathrm{N}$-terminal domain, do not have a significant impact on selective binding into the Cterminal carbohydrate binding domain.

PolyLacNAc, LacdiNAc, and LacNAc are the primary endogenous ligands for vertebrate galectins, and the Thomsen-Friedenreich antigen (Gal $\beta 1$, 3GalNAc $\alpha 1-\mathrm{O}-\mathrm{Ser} /$ Thr) is the putative ligand for interactions such as the cancer cell surface association of Thomsen-Friedenreich-modified Mucin-1 to extracellular galectin-3. As such, these ligands, which are associated with galectin-3-mediated processes, are nonreducing sugars. ${ }^{41-46}$ The binding interaction between galectin-3 and a nonreducing lactose derivative, methyllactose, was explored in order to compare nonreducing and reducing sugar binding. As demonstrated in Figure 3, binding constants for the galectin-3/methyllactose interaction were determined (GST-purified galectin-3). Using eq 1 with $n=1$, a $K_{\mathrm{D}}$ value of $54 \pm 10 \mu \mathrm{M}$ was found, which is slightly lower but quite similar to values observed for the reducing lactoside hemiacetal. This indicates that galectin-3 binding assays using reducing sugars

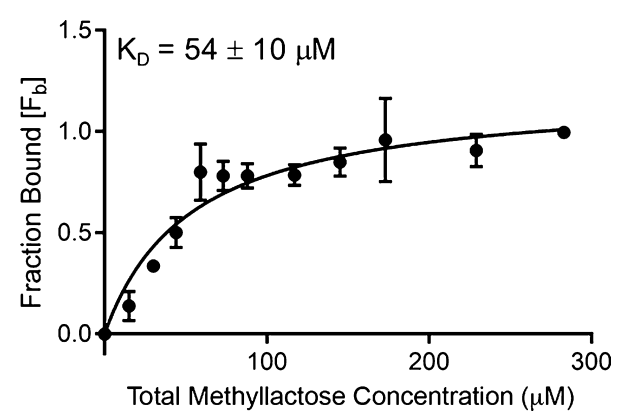

Figure 3. Binding curve for methyllactose and GST-purified galectin3. $B_{\max }=1.20$. The concentration of galectin-3 is $4.3 \mu \mathrm{M}$. Error bars represent the standard deviation arising from triplicate repetitions of the assay. are generally useful despite the fact that the native carbohydrate ligands are often nonreducing.

Lactose-functionalized poly(amidoamine) (PAMAM) dendrimers were synthesized according to our previously published procedure ${ }^{47,48}$ and feature a moderate carbohydrate loading (Figure 4). These multivalent ligands were selected in

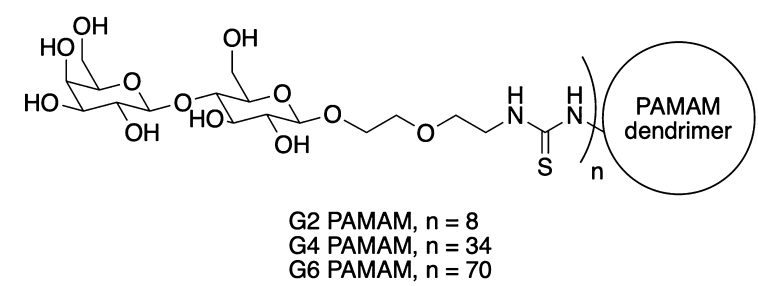

Figure 4. Schematic representation of glycodendrimers used in the reported experiments.

order to observe and quantify the monovalent binding characteristics present within a multivalent system. For these interactions, it is appropriate to calculate binding based on eq 1 in which the Hill coefficient, $n$, is not set to 1 in order to accommodate the multivalent nature of binding.

Comparatively, the glycodendrimer binding affinities are all equivalent with values of $120 \pm 58,100 \pm 45$, and $130 \pm 25$ $\mu \mathrm{M}$ for $\mathrm{G}(2), \mathrm{G}(4)$, and $\mathrm{G}(6)$, respectively. It is important to note that the $n$ terms observed for both the $G(2)$ and the $G(6)$ glycodendrimers were less than one $(n=0.63$ and 0.66 , respectively). These Hill coefficients suggest that more than one galectin-3 binds per glycodendrimer. Upon binding of galectin-3 to a lactoside endgroup on the glycodendrimer surface, the remaining lactosides must overcome steric crowding to bind additional galectin-3 proteins. ${ }^{49,50}$ Some lactosides will not be able to access galectin-3 binding sites, causing negative cooperativity from the perspective of the lactoside ligand (Figure 5a,c, Table 1). For G(4), the Hill coefficient, $\mathrm{n}$, was determined to be $1.0 \pm 0.2$ which is consistent with noncooperative binding. This deviation from the other glycodendrimers can be explained through the optimal flexibility and available surface area of G(4), as compared to $G(2)$ and $G(6)$, which allows for steric hindrance of lactoside dendrimer endgroups to play a less influential role in subsequent binding interactions. ${ }^{51-54}$ Table 1 summarizes the reported binding and cooperativity constants.

As demonstrated in Figure 6a, the net intensity of the intrinsic galectin-3 fluorescence is not significantly changed by binding of lactose. Steady-state fluorescence measurements report the average number of emitted photons over a relatively large period of time (milliseconds or seconds) wherein the 

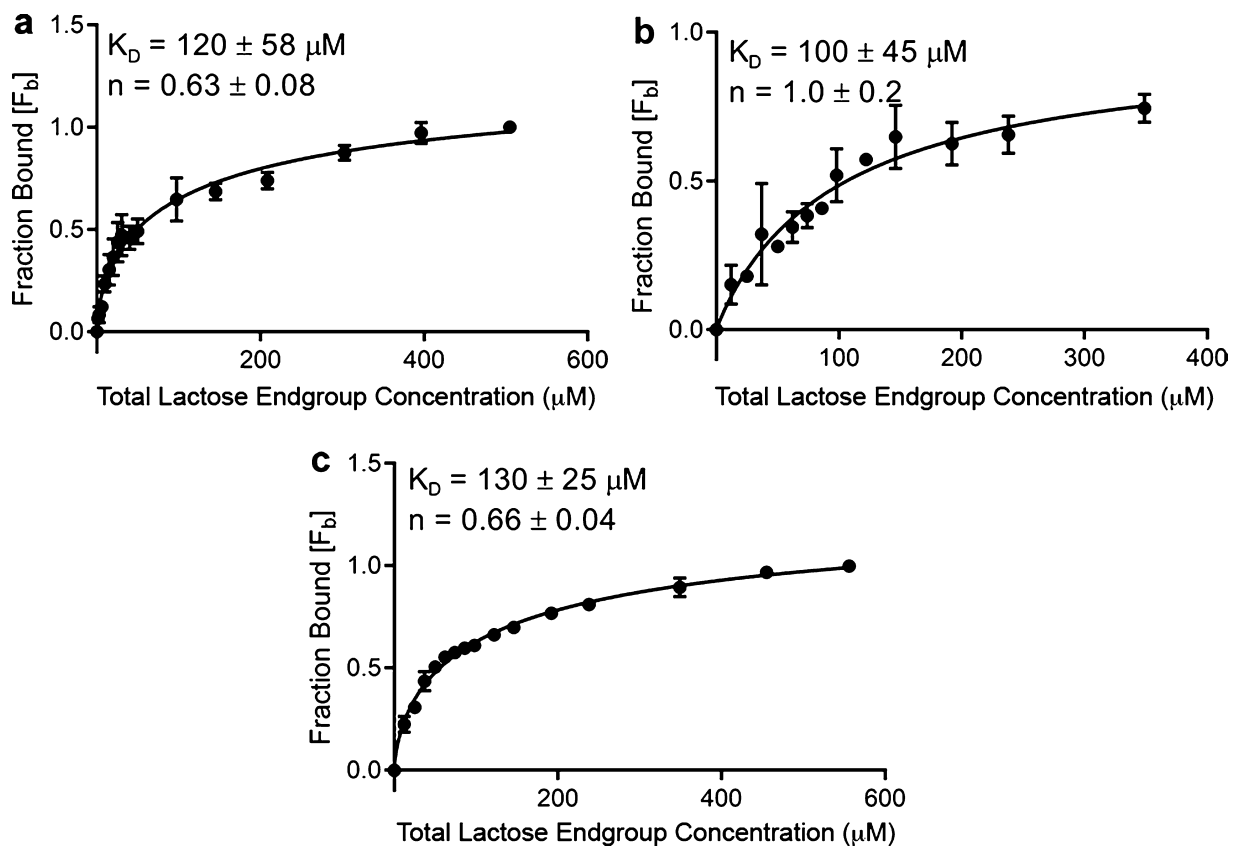

Figure 5. Binding curve for galectin-3 with (a) lactose-functionalized G(2)-PAMAM ( $\left.B_{\max }=1.37\right)$, (b) lactose-functionalized G(4)-PAMAM ( $B_{\max }$ $=0.96)$, and (c) lactose-functionalized G(6)-PAMAM $\left(B_{\max }=1.36\right)$. The concentration of galectin-3 is $4.3 \mu \mathrm{M}$. Error bars represent the standard deviation arising from triplicate repetitions of the assay.

Table 1. Dissociation Constants for Galectin-3 with Monosaccharides and Glycodendrimers

\begin{tabular}{lcc}
\multicolumn{1}{c}{ compound } & $K_{\mathrm{D}}(\mu \mathrm{M})$ & Hill coefficient $(n)$ \\
lactose $^{a}$ & $73 \pm 4$ & $1^{c}$ \\
lactose $^{b}$ & $56 \pm 8$ & $1^{c}$ \\
methyllactose $^{a}$ & $54 \pm 10$ & $1^{c}$ \\
${\text { lactose funct. } \mathrm{G}(2)^{a}}^{a}$ & $120 \pm 58$ & $0.63 \pm 0.08$ \\
lactose funct. $\mathrm{G}(4)^{a}$ & $100 \pm 45$ & $1.0 \pm 0.2$ \\
lactose funct. $\mathrm{G}(6)^{a}$ & $130 \pm 25$ & $0.66 \pm 0.04$
\end{tabular}

${ }^{a}$ GST-purified galectin-3. ${ }^{b}$ His-tagged galectin-3. ${ }^{c}$ Calculation performed with $n=1$.

fluorescing system has achieved an equilibrium. In Figure 6a, steady-state measurements were taken following excitation with $295 \mathrm{~nm}$ light, with the fluorescence emission observed from 300 to $700 \mathrm{~nm}$. The similarity in energy between the excitation and emission wavelengths results in only a small segment of the intrinsic galectin-3 fluorescence emission peak $(>330 \mathrm{~nm})$ being obtainable without saturation. The lack of a significant change in the fluorescence emission spectrum upon the addition of increasing concentrations of lactose and the limited useful range of the emission spectra showcase the need for fluorescence lifetime measurements.

Plotting the normalized waveform differences for galectin-3 fluorescence upon binding of lactose further demonstrates that fluorescence intensities are insufficient for quantification of galectin-3 binding (Figure 6b). Specifically, an increase in intensity in the 17-25 ns time regime and an equivalent decrease in intensity from 25 to $60 \mathrm{~nm}$ suggests that the rate of fluorescence emission is changed by binding, but the overall intensity is not. It is important to note that the differences shown in Figure $6 \mathrm{~b}$ are very small relative to the amplitude of
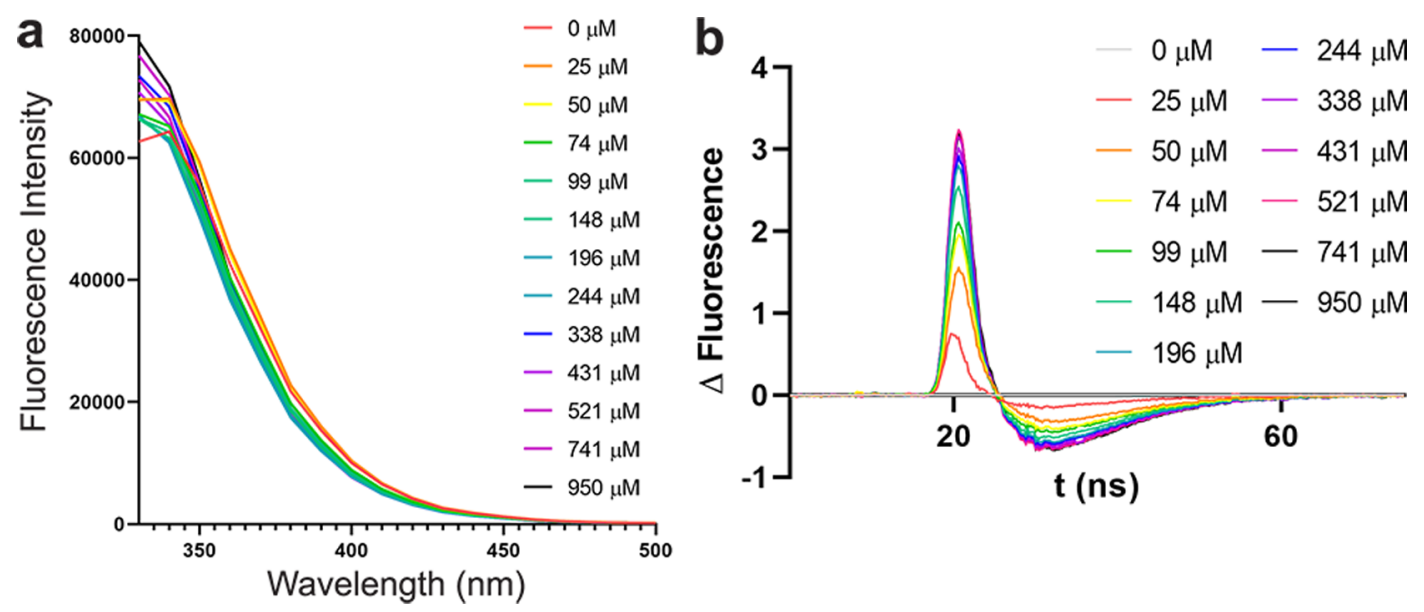

Figure 6. (a) Emission wavelength intensities resulting from excitation at $295 \mathrm{~nm}$. (b) Changes in the fluorescence waveform caused by increasing concentrations of lactose. 
the corresponding waveforms, which are shown in the Supporting Information.

Despite the limited range of emission spectra, binding constants were quantified for the steady-state method. Using eq 1 with $n=1$, a $K_{\mathrm{D}}$ of $167 \pm 18 \mu \mathrm{M}$ was determined. The limited sensitivity of the steady-state measurements and the corresponding large error bars at low concentrations of lactose likely cause the discrepancy in binding constants obtained using steady-state experiments as compared to values obtained using fluorescence lifetime measurements. For both steadystate and lifetime protocols, control experiments were carried out to ensure that fluorescence was not altered by PBS additions, ruling out concentration effects, or by ligand additions independent of galectin-3, ruling out the introduction of unintended fluorophores.

\section{CONCLUSIONS}

The use of time-dependent fluorescence spectroscopy as a quantitative method for determining dissociation constants of difficult to study receptor-ligand interactions is demonstrated. Binding studies with low-affinity monomeric ligands and proteins that are prone to oligomerization are readily obtainable even when only small changes in the fluorescence emission spectra occur upon ligand binding. Improved accuracy was achieved relative to steady-state fluorescence binding studies, and the fluorescence lifetime method of measuring binding constants can provide benchmark values for assessment of other techniques such as surface-based and steady-state studies.

We showcased the utility of this fluorescence lifetime binding methodology by studying carbohydrate binding to galectin-3. Galectin-3 oligomerizes at higher concentrations and in the presence of multivalent ligands, and the monomeric ligand-receptor binding interaction is relatively weak. For these reasons, binding studies are challenging, and a wide range of binding constants has been reported for the interaction of lactose with galectin-3. Time-dependent fluorescence spectroscopy allows accurate determination of solution binding constants for the binding of lactose by galectin-3 (56-73 $\mu \mathrm{M}$, depending on the exact nature of galectin-3 that was used). Comparisons of galectin-3/lactose binding constants to those of methyllactose (because native galectin-3 binds to nonreducing sugars) are reported. In addition, the binding constants for the ligands on a multivalent glycopolymer binding to galectin-3 were reported and were observed to be impacted by receptor crowding effects. Fluorescence lifetime measurements of binding interactions were demonstrated to work well for a challenging, low-affinity system and are be expected to be even more facile for higher affinity, easier to handle ligand-receptor associations.

\section{EXPERIMENTAL SECTION}

General Methods and Reagents. General reagents were purchased from Sigma-Aldrich and Fischer chemical companies, and $\beta$-D-lactose $(80 \% \beta)$ was purchased from Acros Organics. These were used without any further purification. Methods to synthesize methyllactose are described in the Supporting Information. Glycodendrimers were prepared according to the previously published procedure. ${ }^{47,48}$ Dialysis was carried out using Spectra/Por 6-regenerated cellulose tubing obtained from Spectrum Labs (Repligen Corp., Waltham, MA). ${ }^{1} \mathrm{H}$ and ${ }^{13} \mathrm{C}$ NMR spectra were recorded on a Bruker DPX 300 (300 and $75 \mathrm{MHz}$ for ${ }^{1} \mathrm{H}$ and ${ }^{13} \mathrm{C}$, respectively), a Bruker DRX 500 (500 and $126 \mathrm{MHz}$ for ${ }^{1} \mathrm{H}$ and ${ }^{13} \mathrm{C}$, respectively), and an AVANCE III Bruker 600 (600 and $151 \mathrm{MHz}$ for ${ }^{1} \mathrm{H}$ and ${ }^{13} \mathrm{C}$, respectively). Mass spectrometry was carried out on a Bruker Autoflex MALDI-TOF using the indole acrylic acid (IAA) matrix. Dynamic light scattering (DLS) data were collected with a Möbius DLS instrument from Wyatt Technologies (Santa Barbara, CA), proteins were in PBS $(1 \times$ PBS diluted from the Cold Spring Harbor $10 \times$ PBS protocol). Fluorescence lifetime measurements were taken with a UV NovaFluor fluorescence lifetime spectrophotometer from Fluorescence Innovations.

GST-Tagged Galectin-3. Glutathione S-transferase (GST)-tagged galectin-3 was expressed in Escherichia coli transformed with the pGEX-6p-Galectin-3 vector, purified, and the GST tag removed as previously described. ${ }^{55}$ Prior to use, filtration through $0.02 \mu \mathrm{m}$ filters (Whatman Anotop 25) and analysis with Wyatt Mobius DLS were carried out to ensure that galectin-3 oligomers were not present. ${ }^{15}$ The concentration of galectin-3 was determined spectrophotometrically (280 nm, PBS reference) with a molar extinction coefficient of $35,870 \mathrm{M}^{-1} \mathrm{~cm}^{-1}$.

His-Tagged Galectin-3. Cloning. Human galectin-3 was amplified by PCR using the pGEX-6p-Galectin-3 vector as a template. The forward and reverse primers, respectively, were 5'-GGGGACAAGTTTGTACAAAAAAGCAGGCTTCGAAGATAGAACCATGCATCACCATCACCATCACGCAGACAATTTTTCGCTCCAT-3' and 5'-GGGGACCACTTTGTACAAGAAAGCTGGGTCCTATTATATCATGGTATATGAAGCAC- 3 '. The cloning strategy ${ }^{56}$ introduced attB sites and a Shine-Dalgarno sequence, and effectively replaced the Nterminal methionine residue with a Met- $\mathrm{His}_{6}$ sequence to give a minimal $\mathrm{N}$-terminal $6 \times$ His-tagged construct (Met- $-\mathrm{His}_{6}$ fused to residues $2-250$ of human galectin-3). The amplified product was inserted into pDONR201 (Invitrogen) by sitespecific recombination using the Gateway BP clonase reaction following the manufacturer's instructions. The sequenceverified construct was then transferred into pDEST14 (Invitrogen), again using Gateway site-directed recombination, yielding the expression vector pDest14-hGal3-1-250-NHis. Theoretical values for the gene product are a mass of $26,975.18$ $\mathrm{Da}$ and $\mathrm{a} \mathrm{pI}=8.58$, with an extinction coefficient of 35,870 $\mathrm{M}^{-1} \mathrm{~cm}^{-1}$.

Expression and Purification of His-Tagged Galectin-3. For protein expression, E. coli BL21 (DE3)-pRIL cells (Stratagene) were transformed with pDEST14-hGal3-1-250-NHis and plated on LB-agar plates containing $100 \mu \mathrm{g} / \mathrm{mL}$ ampicillin and $25 \mu \mathrm{g} / \mathrm{mL}$ chloramphenicol. A single colony was used to inoculate a $5 \mathrm{~mL}$ overnight $\mathrm{LB}$ starter culture $(100 \mu \mathrm{g} / \mathrm{mL}$ ampicillin and $25 \mu \mathrm{g} / \mathrm{mL}$ chloramphenicol), which was subsequently used to inoculate ZYP-5052-rich autoinduction media $^{57}(1: 1000)$. The culture was shaken with rotation at 250 $\mathrm{rpm}$ at $37^{\circ} \mathrm{C}$ for $18 \mathrm{~h}$. Cells were harvested by centrifugation at $4000 \mathrm{~g}$ for $30 \mathrm{~min}$, and pellets were stored at $-20{ }^{\circ} \mathrm{C}$ until needed. For purification, cell pellets were thawed and resuspended in $5 \mathrm{~mL}$ lysis buffer ( $50 \mathrm{mM}$ Tris- $\mathrm{Cl} \mathrm{pH} 8.0$, $300 \mathrm{mM} \mathrm{NaCl}$, and $1 \mathrm{mM}$ PMSF) per gram of the cell pellet and lysed by French Press. The lysate was centrifuged at $25,000 \mathrm{~g}$ for $30 \mathrm{~min}$, and the supernatant was applied to a NiNTA affinity column. The column was washed with lysis buffer and eluted with lysis buffer and $300 \mathrm{mM}$ imidazole. Fractions containing galectin-3 were combined and applied to a Superdex S-75 size exclusion column equilibrated with PBS. 
Galectin-3 was eluted as a monomer. Protein concentrations were determined by Bradford assay ${ }^{58}$ using the protein assay reagent (Bio-Rad) and BSA as a standard. The purity and molecular weight of galectin-3 were confirmed by SDS-PAGE. Yields were approximately $20 \mathrm{mg}$ of pure galectin-3 per liter of cell culture. Fractions containing pure galectin-3 were combined, and $1 \mathrm{~mL}$ aliquots at $0.5 \mathrm{mg} / \mathrm{mL}$ were stored at $-80{ }^{\circ} \mathrm{C}$.

Fluorescence Lifetime Studies. Fluorescence was monitored using a UV NovaFluor fluorescence lifetime spectrophotometer equipped with a high power $(>20 \mu \mathrm{J}) 532 \mathrm{~nm}$ passively Q-switched laser (Teem photonics). This serves as a pump for a pyrromethane dye $(600 \mu \mathrm{M}$ in ethanol) to create a $560-610 \mathrm{~nm}$ lasing output, which is then passed through a doubling crystal and the subsequent bandpass filter (Semrock) to achieve 285-310 $\mathrm{nm}$ excitation $(1 \mu \mathrm{J} /$ pulse). Samples were excited at $295 \mathrm{~nm}$ to selectively excite tryptophan residues within the protein. Emitted light was selected using a monochromator, and fluorescence decay curves were recorded using direct waveform recording technology.

The concentration of galectin-3 was adjusted by either dilution with PBS or using a protein spin concentrator (Pierce, $10 \mathrm{kDa}$ MWCO) to afford an appropriate concentration (approx. $24 \mu \mathrm{M}, \mathrm{A}_{280}=0.81$ ) stock solution for experimentation. Galectin-3 samples were prepared by combining the appropriate volumes of galectin-3 stock $(24 \mu \mathrm{M})$ and PBS to achieve a $200 \mu \mathrm{L}$ solution at a galectin-3 concentration of 4.3 $\mu \mathrm{M}$ in three wells of a 96-well quartz microplate (Hellma, 730.009-QG). Fluorescence lifetime waveforms were measured in triplicate for galectin-3 solutions at a $0 \mu \mathrm{M}$ ligand concentration and following each ligand addition. Sugar or glycodendrimer stock solutions were made up and introduced to the protein solution in a stepwise manner. GST-purified galectin-3 was used with lactose, methyllactose, and glycodendrimer experiments, while the His-tagged galectin-3 was used with lactose experiments. Specifically, from a $10.0 \mathrm{mM}$ lactose solution in PBS, small aliquots $(4 \times 0.5 \mu \mathrm{L})$ were introduced, followed by increasingly larger volumes $(3 \times 1 \mu \mathrm{L}, 3 \times 2 \mu \mathrm{L}$, and $2 \times 5 \mu \mathrm{L}$ ). These sequential additions resulted in lactose concentrations of $25,50,74,99,148,196,244,338,431$, and $521 \mu \mathrm{M}$. For a single experiment, analysis was completed in three wells. Galectin-3 fluorescence waveforms were obtained in triplicate for individual wells and averaged for each concentration. Values of $F_{b}$ (eq 1) for each concentration were the coefficients obtained by fitting each waveform to a linear combination of unbound $([L]=0 \mu \mathrm{M})$ and saturated galectin- 3 waveforms. $F_{b}$ values, reported as the y-coordinates in ligand binding curves, are the average of individual well coefficients for replicates within an experiment, and the corresponding error bars are the standard deviation. For the purposes of demonstrating experimental reproducibility, lactose binding interactions have been determined using both GST-purified and His-tagged galectin-3. Moreover, this procedure afforded the same $K_{\mathrm{D}}$ values across multiple stock solutions, including those made with galectin- 3 from different rounds of expression and purification. The experiment can be performed using the serial dilutions described above or using the desired concentrations in multiple wells.

Steady-State Fluorescence. Fluorescence was monitored using a BioTek Synergy H1 microplate reader. Galectin-3 excitation was achieved at $295 \mathrm{~nm}$, and emission was monitored from 300 to $700 \mathrm{~nm}$.
Galectin-3 samples were prepared by combining the appropriate volumes of galectin-3 stock $(24 \mu \mathrm{M})$ and PBS to achieve a $200 \mu \mathrm{L}$ solution at a galectin- 3 concentration of 4.3 $\mu \mathrm{M}$ in three wells of a 96-well quartz microplate (Hellma, 730.009-QG). Fluorescence emission spectra were measured for galectin-3 solutions at a $0 \mu \mathrm{M}$ ligand concentration and following each ligand addition. From a $10.0 \mathrm{mM}$ lactose solution in PBS, small aliquots $(4 \times 0.5 \mu \mathrm{L})$ were introduced, followed by increasingly larger volumes $(3 \times 1 \mu \mathrm{L}, 3 \times 2 \mu \mathrm{L}$, and $2 \times 5 \mu \mathrm{L})$. These sequential additions resulted in lactose concentrations of $25,50,74,99,148,196,244,338,431,521$, 741 , and $950 \mu \mathrm{M}$.

\section{ASSOCIATED CONTENT}

\section{Supporting Information}

The Supporting Information is available free of charge at https://pubs.acs.org/doi/10.1021/acsomega.0c03416.

Synthesis procedures and NMR spectra for methyllactose; additional figures for steady-state fluorescence binding experiments; and full description of binding constant calculations (PDF)

Data with embedded formulas; template for calculating binding data (XLSX)

$\mathrm{B}_{\max }$ values and values used for binding calculations (XLSX)

\section{AUTHOR INFORMATION}

\section{Corresponding Author}

Mary J. Cloninger - Department of Chemistry and

Biochemistry, Montana State University, Bozeman, Montana 59718, United States; (1) orcid.org/0000-0002-7551-1003;

Email: mcloninger@montana.edu

\section{Authors}

Samuel P. Bernhard - Department of Chemistry and Biochemistry, Montana State University, Bozeman, Montana 59718, United States

Candace K. Goodman - Department of Chemistry and Biochemistry, Montana State University, Bozeman, Montana 59718, United States

Erienne G. Norton - Department of Chemistry and Biochemistry, Montana State University, Bozeman, Montana 59718, United States

Daniel G. Alme - Department of Chemistry and Biochemistry, Montana State University, Bozeman, Montana 59718, United States

C. Martin Lawrence - Department of Chemistry and Biochemistry, Montana State University, Bozeman, Montana 59718, United States

Complete contact information is available at:

https://pubs.acs.org/10.1021/acsomega.0c03416

\section{Author Contributions}

The manuscript was written through contributions of all authors.

\section{Notes}

The authors declare no competing financial interest.

\section{ACKNOWLEDGMENTS}

The authors acknowledge financial support from NIGMS 62444 and from the Tamara Joy Henderson Fund. E.G.N. acknowledges financial support from NIGMS P20GM103474. 
The content is solely the responsibility of the authors and does not necessarily represent the official views of the National Institutes of Health.

\section{REFERENCES}

(1) Du, X.; Li, Y.; Xia, Y.-L.; Ai, S.-M.; Liang, J.; Sang, P.; Ji, X.-L.; Liu, S.-Q. Insights into Protein-Ligand Interactions: Mechanisms, Models, and Methods. Int. J. Mol. Sci. 2016, 17, 144.

(2) Cloninger, M. J.; Bilgicer, B.; Li, L.; Mangold, S. L.; Phillips, S. T.; Wolfenden, M. L. Multivalency in Supramolecular Chemistry: From Molecules to Nanomaterials; John Wiley \& Sons Ltd: Chichester, U.K., 2012.

(3) Bewley, C. A.; Shahzad-ul-Hussan, S. Characterizing Carbohydrate-Protein Interactions by Nuclear Magnetic Resonance Spectroscopy. Biopolymers 2013, 99, 796-806.

(4) Lakowicz, J. R. Principles of Fluorescence Spectroscopy, 3rd ed.; Springer: New York, 2006.

(5) Meyer-Almes, F.-J. Kinetic binding assays for the analysis of protein-ligand interactions. Drug Discovery Today: Technol. 2015, 17, $1-8$.

(6) Vivian, J. T.; Callis, P. R. Mechanisms of tryptophan fluorescence shifts in proteins. Biophys. J. 2001, 80, 2093-2109.

(7) Callis, P. R. Binding phenomena and fluorescence quenching. II: Photophysics of aromatic residues and dependence of fluorescence spectra on protein conformation. J. Mol. Struct. 2014, 1077, 22-29.

(8) Schlick, K. H.; Lange, C. K.; Gillispie, G. D.; Cloninger, M. J. Characterization of Protein Aggregation via Intrinsic Fluorescence Lifetime. J. Am. Chem. Soc. 2009, 131, 16608-16609.

(9) Muretta, J. M.; Kyrychenko, A.; Ladokhin, A. S.; Kast, D. J.; Gillispie, G. D.; Thomas, D. D. High-performance time-resolved fluorescence by direct waveform recording. Rev. Sci. Instrum. 2010, 81, 103101.

(10) Sciacchitano, S.; Lavra, L.; Morgante, A.; Ulivieri, A.; Magi, F.; De Francesco, G. P.; Bellotti, C.; Salehi, L. B.; Ricci, A. Galectin-3: One Molecule for an Alphabet of Diseases, from A to Z. Int. J. Mol. Sci. 2018, 19, 379.

(11) Cummings, R. D.; Liu, F. T. Galectins. In Essentials of Glycobiology, 2nd ed.; Varki, A., Cummings, R. D., Esko, J. D., Freeze, H. H., Stanley, P., Bertozzi, C. R., Hart, G. W., Etzler, M. E., Eds.; Cold Spring Harbor Laboratory Press: Cold Spring Harbor (NY), 2009.

(12) Hara, A.; Niwa, M.; Noguchi, K.; Kanayama, T.; Niwa, A.; Matsuo, M.; Hatano, Y.; Tomita, H. Galectin-3 as a Next-Generation Biomarker for Detecting Early Stage of Various Diseases. Biomolecules 2020, 10, 389.

(13) Li, Y.-S.; Li, X.-T.; Yu, L.-G.; Wang, L.; Shi, Z.-Y.; Guo, X.-L. Roles of galectin-3 in metabolic disorders and tumor cell metabolism. Int. J. Biol. Macromol. 2020, 142, 463-473.

(14) Romero, A.; Gabius, H.-J. Galectin-3: is this member of a large family of multifunctional lectins (already) a therapeutic target? Expert Opin. Ther. Targets 2019, 23, 819-828.

(15) Bernhard, S. P.; Fricke, M. S.; Haag, R.; Cloninger, M. J. Protein aggregation nucleated by functionalized dendritic polyglycerols. Polym. Chem. 2020, 11, 3849-3862.

(16) Hsu, D. K.; Zuberi, R. I.; Liu, F. T. Biochemical and Biophysical Characterization of Human Recombinant IGE-binding Protein, an Stype Animal Lectin. J. Biol. Chem. 1992, 267, 14167-14174.

(17) Bachhawat-Sikder, K.; Thomas, C. J.; Surolia, A. Thermodynamic analysis of the binding of galactose and poly- $\mathrm{N}$-acetyllactosamine derivatives to human galectin-3. FEBS Lett. 2001, 500, 75-79.

(18) Stowell, S. R.; Arthur, C. M.; Mehta, P.; Slanina, K. A.; Blixt, O.; Leffler, H.; Smith, D. F.; Cummings, R. D. Galectin-1,-2, and-3 exhibit differential recognition of sialylated glycans and blood group antigens. J. Biol. Chem. 2008, 283, 10109-10123.

(19) Sindrewicz, P.; Li, X.; Yates, E. A.; Turnbull, J. E.; Lian, L.-Y.; $\mathrm{Yu}, \mathrm{L}$-G. Intrinsic tryptophan fluorescence spectroscopy reliably determines galectin-ligand interactions. Sci. Rep. 2019, 9, 11851.
(20) Miller, M. C.; Ippel, H.; Suylen, D.; Klyosov, A. A.; Traber, P. G.; Hackeng, T.; Mayo, K. H. Binding of polysaccharides to human galectin-3 at a noncanonical site in its carbohydrate recognition domain. Glycobiology 2016, 26, 88-99.

(21) Ideo, H.; Seko, A.; Ohkura, T.; Matta, K. L.; Yamashita, K. High-affinity binding of recombinant human galectin-4 to SO3-> 3Gal beta 1 -> 3GalNAc pyranoside. Glycobiology 2002, 12, 199-208.

(22) Vrasidas, I.; André, S.; Valentini, P.; Böck, C.; Lensch, M.; Kaltner, H.; Liskamp, R. M. J.; Gabius, H.-J.; Pieters, R. J. Rigidified multivalent lactose molecules and their interactions with mammalian galectins; a route to selective inhibitors. Org. Biomol. Chem. 2003, 1, 803-810.

(23) Tejler, J.; Leffler, H.; Nilsson, U. J. Synthesis of O-galactosyl aldoximes as potent LacNAc-mimetic galectin-3 inhibitors. Bioorg. Med. Chem. Lett. 2005, 15, 2343-2345.

(24) Ahmad, N.; Gabius, H. J.; Sabesan, S.; Oscarson, S.; Brewer, C. F. Thermodynamic binding studies of bivalent oligosaccharides to galectin-1, galectin-3, and the carbohydrate recognition domain of galectin-3. Glycobiology 2004, 14, 817-825.

(25) Brewer, C. F. Thermodynamic binding studies of galectin-1,-3 and-7. Glycoconjugate J. 2002, 19, 459-465.

(26) Sörme, P.; Arnoux, P.; Kahl-Knutsson, B.; Leffler, H.; Rini, J. M.; Nilsson, U. J. Structural and Thermodynamic Studies on Cation-П Interactions in Lectin-Ligand Complexes: High-Affinity Galectin-3 Inhibitors through Fine-Tuning of an Arginine-Arene Interaction. J. Am. Chem. Soc. 2005, 127, 1737-1743.

(27) Brown, S.; Gauvin, C. C.; Charbonneau, A. A.; Burman, N.; Lawrence, C. M.. Csx3 is a cyclic oligonucleotide phosphodiesterase associated with type-III CRISPR-Cas that degrades the second messenger cA4. J. Biol. Chem. 2020.

(28) Zetterberg, F. R.; Peterson, K.; Johnsson, R. E.; Brimert, T.; HÅkansson, M.; Logan, D. T.; Leffler, H.; Nilsson, U. J. Monosaccharide Derivatives with Low-Nanomolar Lectin Affinity and High Selectivity Based on Combined Fluorine-Amide, PhenylArginine, Sulfur-pi, and Halogen Bond Interactions. ChemMedChem 2018, 13, 133-137.

(29) Peterson, K.; Kumar, R.; Stenström, O.; Verma, P.; Verma, P. R.; HÅkansson, M.; Kahl-Knutsson, B.; Zetterberg, F.; Leffler, H.; Akke, M.; Logan, D. T.; Nilsson, U. J. Systematic Tuning of Fluorogalectin-3 Interactions Provides Thiodigalactoside Derivatives with Single-Digit nM Affinity and High Selectivity. J. Med. Chem. 2018, 61, 1164-1175.

(30) Klyosov, A. A.; Witczak, Z. J.; Platt, D. Galectins; Wiley: Hoboken, 2008.

(31) Ippel, H.; Miller, M. C.; Vértesy, S.; Zheng, Y.; Cañada, F. J.; Suylen, D.; Umemoto, K.; Romanò, C.; Hackeng, T.; Tai, G.; Leffler, H.; Kopitz, J.; André, S.; Kübler, D.; Jiménez-Barbero, J.; Oscarson, S.; Gabius, H.-J.; Mayo, K. H. Intra- and intermolecular interactions of human galectin-3: assessment by full-assignment-based NMR. Glycobiology 2016, 26, 888-903.

(32) Saraboji, K.; HÅkansson, M.; Genheden, S.; Diehl, C.; Qvist, J.; Weininger, U.; Nilsson, U. J.; Leffler, H.; Ryde, U.; Akke, M.; Logan, D. T. The Carbohydrate-Binding Site in Galectin-3 Is Preorganized To Recognize a Sugarlike Framework of Oxygens: Ultra-HighResolution Structures and Water Dynamics. Biochemistry 2012, 51, 296-306.

(33) Manzoni, F.; Wallerstein, J.; Schrader, T. E.; Ostermann, A.; Coates, L.; Akke, M.; Blakeley, M. P.; Oksanen, E.; Logan, D. T. Elucidation of Hydrogen Bonding Patterns in Ligand-Free, Lactoseand Glycerol-Bound Galectin-3C by Neutron Crystallography to Guide Drug Design. J. Med. Chem. 2018, 61, 4412-4420.

(34) Hill, A. V. The combinations of haemoglobin with oxygen and with carbon monoxide. I. Biochem. J. 1913, 7, 471-480.

(35) GraphPad Software, Specific Binding with Hill slope. graphpad. com. Accessed Sept. 1, 2020.

(36) Hunter, C. A.; Anderson, H. L. What is Cooperativity? Angew. Chem., Int. Ed. 2009, 48, 7488-7499.

(37) Flores-Ibarra, A.; Vertesy, S.; Medrano, F. J.; Gabius, H.-J.; Romero, A. Crystallization of a human galectin-3 variant with two 
ordered segments in the shortened N-terminal tail. Sci. Rep. 2018, 8, 9835.

(38) Newlaczyl, A. U.; Yu, L.-G. Galectin-3-A jack-of-all-trades in cancer. Cancer Lett. 2011, 313, 123-128.

(39) Ahmad, N.; Gabius, H.-J.; André, S.; Kaltner, H.; Sabesan, S.; Roy, R.; Liu, B.; Macaluso, F.; Brewer, C. F. Galectin-3 precipitates as a pentamer with synthetic multivalent carbohydrates and forms heterogeneous cross-linked complexes. J. Biol. Chem. 2004, 279, 10841-10847.

(40) Johannes, L.; Jacob, R.; Leffler, H. Galectins at a glance. J. Cell Sci. 2018, 131, jcs218826.

(41) Zhao, Q. C.; Barclay, M.; Hilkens, J.; Guo, X. L.; Barrow, H.; Rhodes, J. M.; Yu, L. G. Interaction between circulating galectin-3 and cancer-associated MUC1 enhances tumour cell homotypic aggregation and prevents anoikis. Mol. Cancer 2010, 9, 154.

(42) Kölbl, A. C.; Jeschke, U.; Friese, K.; Andergassen, U. The role of TF- and Tn-antigens in breast cancer metastasis. Histol. Histopathol. 2016, 31, 613-621.

(43) Laaf, D.; Bojarová, P.; Elling, L.; Křen, V. GalectinCarbohydrate Interactions in Biomedicine and Biotechnology. Trends Biotechnol. 2019, 37, 402-415.

(44) Ennist, J. H.; Termuehlen, H. R.; Bernhard, S. P.; Fricke, M. S.; Cloninger, M. J. Chemoenzymatic Synthesis of Galectin Binding Glycopolymers. Bioconjugate Chem. 2018, 29, 4030-4039.

(45) Laaf, D.; Bojarová, P.; Pelantová, H.; Křen, V.; Elling, L. Tailored Multivalent Neo-Glycoproteins: Synthesis, Evaluation, and Application of a Library of Galectin-3-Binding Glycan Ligands. Bioconjugate Chem. 2017, 28, 2832-2840.

(46) Yang, R.-Y.; Rabinovich, G. A.; Liu, F.-T. Galectins: structure, function and therapeutic potential. Expert Rev. Mol. Med. 2008, 10, No. e17.

(47) Goodman, C. K.; Wolfenden, M. L.; Nangia-Makker, P.; Michel, A. K.; Raz, A.; Cloninger, M. J. Multivalent scaffolds induce galectin-3 aggregation into nanoparticles. Beilstein J. Org. Chem. 2014, $10,1570-1577$.

(48) Wolfenden, M.; Cousin, J.; Nangia-Makker, P.; Raz, A.; Cloninger, M. Glycodendrimers and Modified ELISAs: Tools to Elucidate Multivalent Interactions of Galectins 1 and 3. Molecules 2015, 20, 7059-7096.

(49) Hlavacek, W. S.; Posner, R. G.; Perelson, A. S. Steric effects on multivalent ligand-receptor binding: Exclusion of ligand sites by bound cell surface receptors. Biophys. J. 1999, 76, 3031-3043.

(50) Wolfenden, M. L.; Cloninger, M. J. Carbohydrate-functionalized dendrimers to investigate the predictable tunability of multivalent interactions. Bioconjugate Chem. 2006, 17, 958-966.

(51) Pavan, G. M.; Albertazzi, L.; Danani, A. Ability to Adapt: Different Generations of PAMAM Dendrimers Show Different Behaviors in Binding siRNA. J. Phys. Chem. B 2010, 114, 2667-2675.

(52) Kulczynska, A.; Frost, T.; Margerum, L. D. Effect of PAMAM dendrimer size and $\mathrm{pH}$ on the electrostatic binding of metal complexes using cyclic voltammetry. Macromolecules 2006, 39, $7372-7377$

(53) Jockusch, S.; Ramirez, J.; Sanghvi, K.; Nociti, R.; Turro, N. J.; Tomalia, D. A. Comparison of nitrogen core and ethylenediamine core starburst dendrimers through photochemical and spectroscopic probes. Macromolecules 1999, 32, 4419-4423.

(54) Maiti, P. K.; Çağın, T.; Wang, G.; Goddard, W. A. Structure of PAMAM dendrimers: Generations 1 through 11. Macromolecules 2004, 37, 6236-6254.

(55) Nangia-Makker, P.; Balan, V.; Raz, A. Galectin-3 Binding and Metastasis. In Metastasis Research Protocols; Dwek, M., Brooks, S. A., Schumacher, U., Eds.; Humana Press: Totowa, NJ, 2012; pp 251266.

(56) Menon, S. K.; Eilers, B. J.; Young, M. J.; Lawrence, C. M. The crystal structure of D212 from sulfolobus spindle-shaped virus ragged hills reveals a new member of the PD-(D/E)XK nuclease superfamily. J. Virol. 2010, 84, 5890-5897.

(57) Studier, F. W. Protein production by auto-induction in high density shaking cultures. Protein Expression Purif. 2005, 41, 207.
(58) Bradford, M. M. A rapid and sensitive method for the quantitation of microgram quantities of protein utilizing the principle of protein-dye binding. Anal. Biochem. 1976, 72, 248-254. 